LPTENS 02/63

hep-th/0212217

\title{
Relativistic String in a Pulse
}

\author{
Constantin Bachas \\ LPTENS, 24 rue Lhomond, 75231 Paris cedex 05, France ${ }^{1}$
}

\begin{abstract}
I study a relativistic open string coupling through its endpoints to a plane wave with arbitrary temporal profile. The string's transverse oscillations respond linearly to the external field. This makes it possible to solve the classical equations, and to calculate the quantum-mechanical S-matrix in closed form. I analyze the dynamics of the string as the characteristic frequency and duration of the pulse are continuously varied. I derive, in particular, the multipole expansion in the adiabatic limit of very long wavelengths, and discuss also more violent phenomena such as shock waves, cusps and null brane intersections. Apart from their relevance to the study of time-dependence in superstring theory, these results could have other applications, such as the teleportation of gravitational wave bursts by cosmic strings.
\end{abstract}

1 Laboratoire mixte du CNRS et de l' Ecole Normale Supérieure 


\section{Introduction and Outlook}

Many authors have considered relativistic strings moving in the background of a planefronted wave [1-25]. The original motivations were the study of the high-energy scattering of fundamental strings [26][1], and of the fate of null spacetime singularities [3][4]. More recently, interest in pp-wave backgrounds has been mainly spurred by their conjectured holographic relation to limits of supersymmetric gauge theories [7], and because they provide simple models of time dependence in string theory. Finally, the interactions of cosmic strings $^{2}$ with electromagnetic and gravitational waves may have played an important role in primordial cosmology, and can be the source of detectable gravity waves today.

A plane-fronted gravitational wave is described in Brinkmann coordinates by the following metric and curvature tensor :

$$
d s^{2}=-2 d x^{+} d x^{-}+d \mathbf{x} d \mathbf{x}+H\left(x^{+}, \mathbf{x}\right)\left(d x^{+}\right)^{2} \quad \text { and } \quad R_{i+j+}=\partial_{i} \partial_{j} H
$$

To study a relativistic string in this background, it is convenient to go to the light-cone gauge, $X^{+}=p^{+} \tau$, in which $H\left(p^{+} \tau, \mathbf{X}\right)$ is a time-dependent potential for the transverse coordinates. The dynamics simplifies greatly in the case of waves with planar symmetry, also called exact plane waves [28], for which $R_{i+j+}$ is constant on the wavefronts. In this case $H=A_{i j}\left(x^{+}\right) X^{i} X^{j}$, so that the $\mathbf{X}(\sigma, \tau)$ are free worldsheet fields, with a mass matrix $A_{i j}$ that depends only on time. Even this simple problem is, however, still too hard to solve, except for special time profiles [3][6][8][25] including of course the maximally supersymmetric cases [29] where $A_{i j}$ is constant. The difficulty can be essentially traced to the fact that, although the $\mathbf{X}(\sigma, \tau)$ obey a linear equation, they have a highly non-local and non-linear dependence on the wave profile.

In this paper I show that the problem simplifies further for an open string that couples to a plane wave only through its endpoints. Such waves are electromagnetic rather than gravitational, or they may correspond to geometric vibrations of D-branes. The striking fact about them is that they enter as a source term in the field equations for $\mathbf{X}(\sigma, \tau)$, so that transverse string oscillations respond linearly to the incident wave. This means that one can solve the classical equations, and compute the exact quantum mechanical S-matrix, for any temporal profile of the incident pulse. We will thus study the dynamics of the string as the characteristic frequency and duration of the pulse are continuously varied. I will

2 For a review of cosmic strings see [27]. 
show, in particular, how the process of string excitation, invisible in the long-wavelength multipole expansion, becomes the dominant effect for characteristic frequencies of the order of $\left(\alpha^{\prime} p^{+}\right)^{-1}$, where $\alpha^{\prime}$ is the Regge slope and $p^{+}$the conserved light-cone momentum.

Because of their great simplicity, these plane waves are privileged backgrounds for the study of time dependence in string theory. In contrast to other recent works, where open strings have been attached to D-branes in a gravitational pp-wave [9][10][12][13][15][18], here the worldsheet theory is both massless and free. Furthermore, the freedom to choose the wave profile makes it possible to regularize both the ultraviolet and the infrared, so that the first quantized theory has a well defined, albeit non-trivial S-matrix. This opens the way for addressing a host of other interesting questions : what are the effects of backreaction, and of open- and closed-string radiation, when string interactions are turned on? How does the boundary state for an undulating D-brane capture its non-trivial timedependence? What is the holographic description of string theory in the background of a large collection of undulating branes? On a different register, does the linearity of the coupling to D-brane plane waves impose constraints on the non-abelian extension of the Dirac-Born-Infeld action? And finally, what can we learn about high-energy scattering, and about the fate of cosmological singularities ${ }^{3}$ in string theory? The fact that the worldhseet theory is massless and free should make some of these questions quite tractable. I hope to return to them in a forthcoming publication.

Apart from their relevance to superstring theory, the results of this paper could be also of interest in the context of large cosmic strings. As I will argue later, in section 8, the simplified model studied here could be relevant for a cosmic string passing near a source of strong gravitational waves. The coherent excitation of the string may result in a teleportation of the signal, assuming radiation damping can be neglected. This question deserves, as I will explain, further investigation.

The plan of the paper is as follows :

- Section 2 introduces the basic backgrounds, which are parallel D-branes with plane Born-Infeld or vibration waves on their worldvolumes. I explain why these are exact supersymmetric backgrounds of superstring theory with arbitrary temporal profiles, and compute their total momentum and energy.

3 My interest in these backgrounds has been motivated by the analogy between null brane intersections and orbifold singularities, advocated in ref. [20]. 
- In section 3 we will solve the classical equations of motion for an open string with both endpoints on the same undulating D-brane. I discuss the point-particle limit, and comment on the similarities between plane-fronted gravitational waves and brane waves.

- Section 4 generalizes the solution to open strings with endpoints on distinct Dbranes, or in T-dual language to strings charged with respect to an electromagnetic pulse. The main novel feature in this case, is that the wave can impart momentum to the string, even in the limit of extremely long wavelength. The reason is that the string stretches as the D-branes separate, or, in T-dual language, that charges can accelerate in the field of a plane electromagnetic pulse.

- Section 5 presents the quantization of the open string in the wave background. I calculate the exact S-matrix, paying particular attention to zero modes, and derive the excitation probabilities and average mass squared of a string that was initially in its ground state. The string emerges in a coherent state, dictated by the temporal profile of the pulse.

- Section 6 is devoted to the long-wavelength limit of the classical solution. I derive a systematic multipole expansion in powers of $\alpha^{\prime} p^{+}$, or equivalently of derivatives of the background fields, and show that it describes a string surfing on the wave and emerging behind it adiabatically elongated or contracted. String excitation cannot be seen at any finite order of this multipole expansion.

- In section 7 we will consider the opposite limit of shock waves, and of waves with cusps and kinks. We will see how an incident (primary) short pulse transmits a (secondary) clone pulse on the string, which carries away a fixed fraction of the incident energy and momentum. We will also consider other, potentially violent phenomena, such as resonances and null brane intersections, and comment on the analogy of the latter with null singularities in orbifold models.

- In section 8 we will ask under what conditions coherent string excitation can be observed in nature. I comment on highly energetic cosmic rays in laser beams, and on large cosmic strings near strong sources of gravitational waves. These latter could be relevant to the experimental search for gravity waves.

Let me finally note that to keep the paper short, I focus mainly on the bosonic degrees of freedom of the string in what follows. Incorporating explicitly the fermions does not present great difficulties, and might have obscured our discussion. In the context of cosmic strings, or of effective QCD strings, the fermions are anyway not required. For fundamental strings, on the other hand, supersymmetry will be always implicitly present. It guarantees that the background configuration is stable, and that one can try to define a perturbative S-matrix around it. 


\section{Travelling Waves on a D-brane}

Figure 1 shows a D-string with a wave of arbitrary temporal profile travelling at the speed of light in the (negative) $O 1$ direction. Also shown in the figure is a reference, static and straight D-string. The background spacetime is flat, with coordinates

$$
x^{\mu}=\left(x^{+}, x^{-}, \mathbf{x}\right), \quad \text { where } \quad x^{ \pm}=\frac{x^{0} \pm x^{1}}{\sqrt{2}} \quad \text { and } \quad \mathbf{x} \in \mathbf{R}^{8} .
$$

The D-strings are described in static gauge by the embedding

$$
Y^{ \pm}=\zeta^{ \pm}, \quad \mathbf{Y}=\mathbf{Y}\left(\zeta^{+}\right)
$$

Here, and throughout this paper, we use $Y^{\mu}\left(\zeta^{a}\right)$ to denote the spacetime embedding of a D-brane, and $X^{\mu}(\sigma, \tau)$ for the embedding of a fundamental string. The reference D-string of Figure 1 has $\mathbf{Y}=0$, while for the undulating one $\mathbf{Y} \equiv\left(Y^{2}, \cdots, Y^{9}\right)$ is an arbitrary vector-valued function of $\zeta^{+}$.

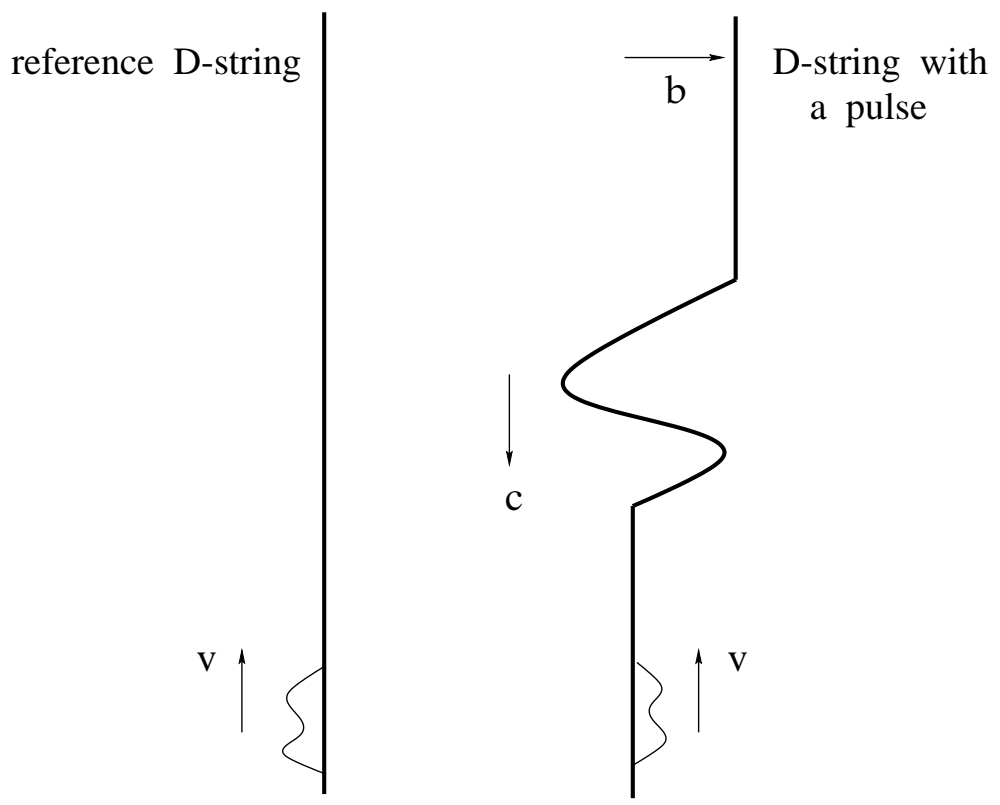

Fig. 1: A static, straight D-string (left) and a D-string with a wave travelling in the downward direction at the speed of light (right). The wiggly lines are open fundamental test strings moving in the opposite, upward direction. Since there is neither dissipation nor dispersion, the pulse keeps its profile and energy at all times. After its passage, the D-string is displaced by $\mathbf{b}$ in the transverse space. 
The undulating D-string is an exact, consistent background of open-string theory for any choice of the shape function $\mathbf{Y}\left(\zeta^{+}\right)$. This may not seem obvious at first, because the effective Dirac-Born-Infeld equations for a D-brane receive $\alpha^{\prime}$ corrections, which generally depend on the extrinsic curvature [30][31]. One can, however, argue directly in conformal field theory that the boundary operator

$$
S_{\text {boundary }}=\int \frac{d \tau}{2 \pi \alpha^{\prime}} \mathbf{Y}\left(X^{+}\right) \cdot \partial_{\sigma} \mathbf{X},
$$

which describes the coupling of the open string to the background wave, is marginal for any choice of the profile function. This is so because the free worldsheet field $X^{+}(\sigma, \tau)$ has vanishing OPEs both with itself, and with the transverse-coordinate fields $\mathbf{X}(\sigma, \tau)$. An alternative but equivalent argument is that all potential contributions to the spacetime equations for $\mathbf{Y}\left(\zeta^{a}\right)$ are zero, because terms having a lower 'minus' index vanish, while those without a minus index cannot be contracted.

Actually the above configuration is not only consistent, but it is also $1 / 4$ supersymmetric. If $\epsilon$ and $\bar{\epsilon}$ are the two Weyl-Majorana supersymmetry parameters of type IIB theory, then the unbroken supersymmetries obey

$$
\Pi \epsilon=\bar{\epsilon} \quad \text { and } \quad \gamma^{+} \epsilon=0,
$$

where $\Pi$ is the product of $\gamma$ matrices along the directions of the reference brane (up to a phase [32]). Supersymmetric strings with travelling waves have been discussed widely in the literature, both in the supergravity approximation [33] , and more recently as consistent configurations of D-branes [34][35][36][37] . They play an important role in the study of string dualities, and in the statistical description of near-extremal black holes.

The wave illustrated in Figure 1 dies out asymptotically, meaning that $\mathbf{Y}$ goes to constant values in the distant future and in the distant past. This guarantees that the pulse has finite energy, and that we can define asymptotic states for the scattering problem. By an appropriate choice of the origin of coordinates we can set

$$
\mathbf{Y}(-\infty)=0 \quad \text { and } \quad \mathbf{Y}(+\infty)=\mathbf{b}
$$

We will relax this condition, and consider infinite wave trains towards the end of this paper. Until further notice (2.5) will be, however, implicitly assumed. 
By the standard rules of T-duality (see for instance [32]) one can map the undulating D-string to an equivalent higher-dimensional D-brane, with a plane electromagnetic wave on its worldvolume,

$$
Y^{j}\left(\zeta^{+}\right) \equiv 2 \pi \alpha^{\prime} A^{j}\left(\zeta^{+}\right)
$$

This is a homogeneous, generally non-monochromatic, electromagnetic wave. In what follows, I will mostly use the geometric language of undulating D-branes, which is easier to visualize. All the results will, however, apply with little change to plane electromagnetic waves interacting with the endpoints of an open string.

To conclude this section, let us calculate the energy and momentum carried by a pulse. To leading order in the $\alpha^{\prime}$ expansion this is given by the variation of the Dirac-NambuGotto action for the D-string,

$$
T^{\mu \nu}(x)=-2 T_{D} \int d^{2} \zeta \frac{\partial \sqrt{-\hat{g}(\zeta)}}{\partial G_{\mu \nu}(x)},
$$

where $\hat{g}$ is the determinant of the induced metric, $T_{D}$ the tension of the D-string, and $G_{\mu \nu}$ the spacetime metric. Using the identity

$$
\frac{\partial \hat{g}(\zeta)}{\partial G_{\mu \nu}(x)}=\hat{g}(\zeta) \hat{g}^{a b} \partial_{a} Y^{\mu} \partial_{b} Y^{\nu} \delta^{(10)}\left(x^{\rho}-Y^{\rho}(\zeta)\right)
$$

and the special form of the induced metric in our problem,

$$
\left(\begin{array}{cc}
\hat{g}_{++} & \hat{g}_{+-} \\
\hat{g}_{-+} & \hat{g}_{--}
\end{array}\right)=\left(\begin{array}{cc}
\partial_{+} \mathbf{Y} \cdot \partial_{+} \mathbf{Y} & -1 \\
-1 & 0
\end{array}\right)
$$

one finds the following non-vanishing components of the energy-momentum tensor

$$
\left.\begin{array}{l}
T^{+-} \\
T^{--} \\
T^{-j}
\end{array}\right\}=T_{D} \delta^{(8)}(\mathbf{x}-\mathbf{Y}(\zeta)) \times\left\{\begin{array}{c}
1 \\
\partial_{+} \mathbf{Y} \cdot \partial_{+} \mathbf{Y} \\
\partial_{+} Y^{j}
\end{array}\right.
$$

Note that the right-hand side is a function of $x^{+}=\zeta^{+}$. Subtracting the energy-momentum tensor of the static reference D-string, and integrating over $\mathbf{R}^{9}$, gives the total energy and momentum of the pulse :

$$
P_{\text {pulse }}^{+}=0 \quad \text { and } \quad P_{\text {pulse }}^{-}=T_{D} \int \frac{d x^{1}}{\sqrt{2}} \partial_{+} \mathbf{Y} \cdot \partial_{+} \mathbf{Y} .
$$

Finiteness of the energy requires that $\mathbf{Y}$ grows at most like $\left|x^{+}\right|^{1 / 2}$ at infinity, a weaker condition than the assumption (2.5) that we already made. 
The semiclassical configuration (2.2) is a coherent superposition of open strings, moving in the downward direction at the speed of light. In terms of the Fourier components of $\mathbf{Y}$,

$$
\mathbf{Y}\left(x^{+}\right) \equiv \int_{-\infty}^{\infty} \frac{d p^{-}}{p^{-}} \mathbf{y}\left(p^{-}\right) e^{i p^{-} x^{+}}
$$

the total energy-momentum of the pulse reads

$$
P_{\text {pulse }}^{-}=4 \pi T_{D} \int_{0}^{\infty} d p^{-}\left|\mathbf{y}\left(p^{-}\right)\right|^{2}
$$

The average density of open strings with momentum $p^{-}$is therefore

$$
p^{-}<n\left(p^{-}\right)>=4 \pi T_{D}\left|\mathbf{y}\left(p^{-}\right)\right|^{2}
$$

Since $T_{D} \sim 1 / g_{s}$, this density is very large at weak string coupling. Fluctuations in the density are subleading, $\delta n \sim o(\sqrt{n})$, consistently with the semiclassical treatment of the pulse. Note that if $\mathbf{Y}$ dies out more slowly than $\sim 1 / x^{+}$at infinity, the average number density of open strings diverges in the infrared.

\section{Neutral Test Particle and String}

We turn next to the analysis of open strings moving in the background of such travelling waves. There are two types of open strings : (a) those illustrated in Figure 1, which have both endpoints on the same D-brane, and (b) those with endpoints on two distinct D-branes (see Figure 2). In the T-dual language, the former correspond to neutral strings, while the latter are charged with respect to the electromagnetic wave. I will consider the neutral case first, and extend the analysis to charged strings in the following section. I will also first solve the classical problem, and then proceed to quantize the string in section 5. The study of the second-quantized theory, and in particular of the backreaction of the string on the travelling wave, is postponed to a future publication.

Before analyzing relativistic strings, it is instructive to first consider a point particle confined to move on the D-string of Figure 1. The net effect of the pulse on the particle is a time delay, and a displacement $\mathbf{b}$ in the transverse space. Nothing else can happen to the particle, since (a) its momentum $p^{+}$is conserved, (b) there are no internal degrees of freedom to excite, and (c) the mass-shell condition $2 p^{+} p^{-}=m^{2}$ implies that $p^{-}$is fixed in 
the asymptotic past and future. To calculate the time delay of the particle, let us define flat coordinates on the D-string,

$$
\zeta^{+}=x^{+} \quad \text { and } \quad \zeta^{-}=x^{-}-\frac{1}{2} \int_{-\infty}^{x^{+}}\left(\partial_{+} \mathbf{Y}\right)^{2}
$$

In these coordinates $\hat{g}_{\alpha \beta}=\eta_{\alpha \beta}$ and the particle's worldline is a straight line $: \zeta^{ \pm}=p^{ \pm} \tau$. Thus, in terms of the original spacetime coordinates we have :

$$
X^{+}(\tau)=p^{+} \tau \quad \text { and } \quad X^{-}(\tau)=p^{-} \tau+\frac{1}{2} \int_{-\infty}^{p^{+} \tau}\left(\partial_{+} \mathbf{Y}\right)^{2}
$$

Comparing with the worldline on a straight reference D-string, for which $X^{ \pm}=p^{ \pm} \tau$, we see that the passage of the wave leads to a net total shift of $X^{-}$by an amount

$$
\delta X^{-}=\frac{1}{2} \int_{-\infty}^{+\infty}\left(\partial_{+} \mathbf{Y}\right)^{2}=\frac{P_{\text {pulse }}^{-}}{2 T_{D}}
$$

The second equality in this expression follows from eq. (2.11) . The positive-definite shift of $X^{-}$implies that the particle on the undulating string reaches any given point far behind the pulse later than if it were moving on the reference brane.

The above results are strongly reminiscent of the physics of gravitational waves. Particles crossing, for example, a gravitational shock wave [38][39] experience a shift $\delta X^{-} \propto G_{N} P^{-}$, reminiscent of the delay (3.3) . The passage of a gravitational wave burst can cause, furthermore, a net transverse displacement, like b, an effect known as the 'memory' of the wave [40][41]. These similarities should not come as a surprise, since our point particle couples to the induced metric (2.9), which has precisely the Brinkmann form of a plane-fronted wave. The reader may here object that in a real gravitational wave $g_{++}$must depend on transverse coordinates, since otherwise $R_{i+j+}=0$ and the spacetime is everywhere flat. The transverse space is, however, effectively 'deconstructed' in our case by juxtaposing several D-strings, each with a different wave profile $\mathbf{Y}\left(x^{+}\right)$. The time delay (3.3) , in particular, arises because we compare motion on the undulating D-string, to motion on a static reference D-brane.

Consider next a relativistic open string in the background of the travelling wave. The embedding coordinates of the open string obey the usual free-wave equation

$$
\left(\partial_{\tau}^{2}-\partial_{\sigma}^{2}\right) X^{\mu}(\sigma, \tau)=0
$$


and Virasoro conditions

$$
\partial_{+} X^{\mu} \partial_{+} X_{\mu}=\partial_{-} X^{\mu} \partial_{-} X_{\mu}=0
$$

where $\partial_{ \pm}=\frac{1}{2}\left(\partial_{\tau} \pm \partial_{\sigma}\right)$. Since the center-of-mass momentum $p^{+}$is conserved, we can go to the lightcone gauge in which

$$
X^{+}=2 \alpha^{\prime} p^{+} \tau \quad \text { and } \quad 2 \alpha^{\prime} p^{+} \partial_{ \pm} X^{-}=\partial_{ \pm} \mathbf{X} \cdot \partial_{ \pm} \mathbf{X}
$$

We set from now on $2 \alpha^{\prime}=1$. As usual, one can solve the Virasoro conditions for $X^{-}(\sigma, \tau)$ in terms of $p^{+}$and of the transverse coordinates $\mathbf{X}(\sigma, \tau)$. The latter must obey the boundary conditions

$$
\mathbf{X}(\sigma, \tau)=\mathbf{Y}\left(p^{+} \tau\right) \text { at } \sigma=0 \text { or } \pi
$$

since both string endpoints lie on the worldline of the undulating D-string. Equations (3.4) and (3.7) define a linear problem, in which the wave profile $\mathbf{Y}\left(p^{+} \tau\right)$ enters as a source. This should be contrasted to other electromagnetic backgrounds [42-44] which, even when they leave the string equations linear, modify at the least their homogeneous part.

The general solution to the above linear problem can be written in two different ways as follows :

$$
\mathbf{X}=\mathbf{X}_{\text {in }}+\delta \mathbf{X}_{\text {in }}=\mathbf{X}_{\text {out }}+\delta \mathbf{X}_{\text {out }}
$$

where $\mathbf{X}_{\text {in (out) }}$ are general solutions to the homogeneous problem long before (or after) the passage of the pulse, and $\delta \mathbf{X}_{\text {in (out) }}$ are special solutions (up to a constant) that vanish at $\tau \rightarrow-\infty(+\infty)$. Since $\mathbf{X}_{\mathrm{in}}$ and $\mathbf{X}_{\text {out }}$ obey standard Dirichlet conditions at both string endpoints, their mode expansions are of the usual form :

$$
\mathbf{X}_{\mathrm{in}}(\sigma, \tau)=\sum_{n \neq 0} \frac{1}{n} \mathbf{a}_{n}^{(\mathrm{in})} e^{-i n \tau} \sin (n \sigma)
$$

and

$$
\mathbf{X}_{\text {out }}(\sigma, \tau)=\mathbf{b}+\sum_{n \neq 0} \frac{1}{n} \mathbf{a}_{n}^{\text {(out) }} e^{-i n \tau} \sin (n \sigma) .
$$

Our main new result is the following exact expression for the special solutions :

$$
\delta \mathbf{X}_{\mathrm{in}}(\sigma, \tau)=\mathbf{F}_{\mathrm{in}}\left(\tau-\sigma, p^{+}\right)+\mathbf{F}_{\mathrm{in}}\left(\tau+\sigma-\pi, p^{+}\right)
$$


and similarly for 'in' replaced by 'out', where the functions $\mathbf{F}_{\text {in (out) }}$ are defined by the following alternating sums :

$$
\mathbf{F}_{\mathrm{in}}(a, \lambda)=\sum_{N=0}^{\infty}(-)^{N} \mathbf{Y}(\lambda a-\lambda N \pi)
$$

and

$$
\mathbf{F}_{\text {out }}(a, \lambda)=-\sum_{N=1}^{\infty}(-)^{N}[\mathbf{Y}(\lambda a+\lambda N \pi)-\mathbf{b}] .
$$

To check that the boundary conditions (3.7) are indeed satisfied, the reader should note that the function defined in (3.12) obeys the relation $\mathbf{F}_{\text {in }}\left(\tau, p^{+}\right)+\mathbf{F}_{\text {in }}\left(\tau-\pi, p^{+}\right)=\mathbf{Y}\left(p^{+} \tau\right)$, and that a similar relation holds for the function $\mathbf{F}_{\text {out }}$. Furthermore, for $p^{+}$strictly positive, $\delta \mathbf{X}_{\mathrm{in}}$ vanishes in the asymptotic past provided that $\mathbf{Y}$ goes to zero fast enough, and the same is true for $\delta \mathbf{X}_{\text {out }}$ in the asymptotic future.

Equations (3.8)-(3.12) are the complete solution to the initial-value problem, whenever the alternating sum $\mathbf{F}_{\text {in }}(a, \lambda)$ can be defined. Note that in the $p^{+} \rightarrow 0$ limit, the solution reduces to a point-like string riding the wave at constant values of $x^{+}$and of $\mathbf{X}=\mathbf{Y}\left(x^{+}\right)$. When $p^{+} \neq 0$ the string collides inevitably with the travelling wave, and will emerge in general with different momentum $p^{-}$and mass $M$. We will analyze this process in detail, for various wave profiles and in different physical contexts, in the following sections. First, however, I will generalize the above solution to the case of an open string stretching between two different D-branes.

\section{Charged Particle and String}

This more general situation is illustrated in Figure 2. The oriented open string has its left endpoint on one D-string, and its right endpoint on another. The travelling waves on these two D-strings are a priori different, and can be decomposed into a synchronized and a relative motion :

$$
\mathbf{Y}_{\text {left }}=\mathbf{Y}\left(x^{+}\right)+\mathbf{Z}\left(x^{+}\right) \quad \text { and } \quad \mathbf{Y}_{\text {right }}=\mathbf{Y}\left(x^{+}\right)-\mathbf{Z}\left(x^{+}\right) .
$$

This configuration is still a consistent, 1/4 supersymmetric background of open-string field theory. The boundary conditions in light-cone gauge are :

$$
\mathbf{X}(0, \tau)=\mathbf{Y}_{\text {left }}\left(p^{+} \tau\right) \text { and } \quad \mathbf{X}(\pi, \tau)=\mathbf{Y}_{\text {right }}\left(p^{+} \tau\right)
$$

Clearly, in the absence of the relative wave the problem reduces to the one of the previous section. Our task here is to generalize the solution to the case of non-trivial $\mathbf{Z}\left(x^{+}\right)$. 


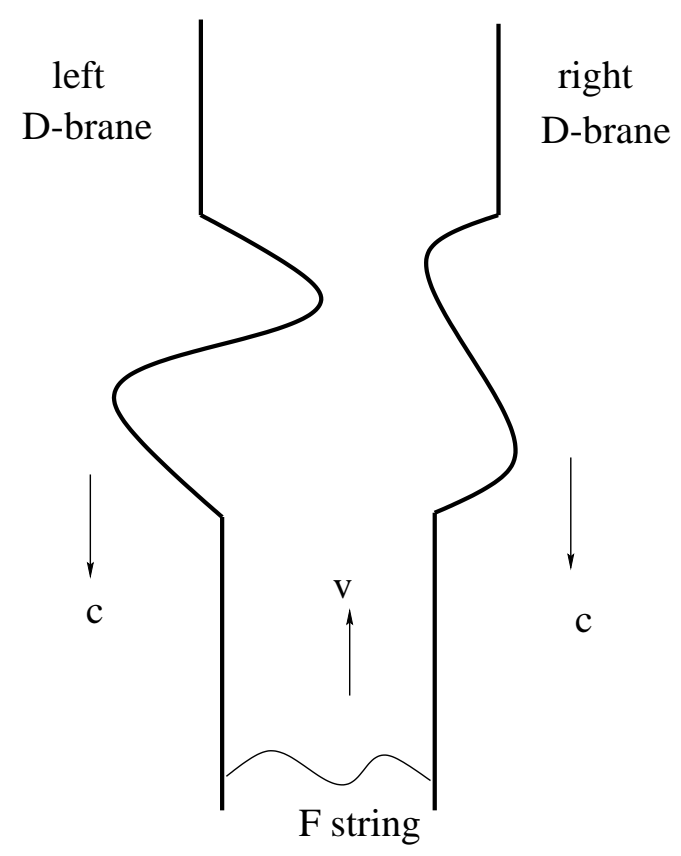

Fig. 2: An open string stretching between two D-branes with different plane-waves on their worldvolumes. The passage of the wave train can change the distance between the two D-branes, and hence also the minimal mass of the string. In the T-dual configuration this corresponds to a charged string picking up transverse momentum in the electric field of the wave.

The general solution can be again written in the form (3.8), where $\mathbf{X}_{\mathrm{in} \text { (out) }}$ are the asymptotic fields that obey conventional Dirichlet boundary conditions, and $\delta \mathbf{X}_{\text {in (out) }}$ vanish in the distant past (or future) as before. To be explicit, let us assume that $\mathbf{Y}$ has the asymptotic limits $(2.5)$, while

$$
\mathbf{Z}(-\infty)=\pi \mathbf{w}_{\text {in }} \quad \text { and } \quad \mathbf{Z}(+\infty)=\pi \mathbf{w}_{\text {out }},
$$

so that the passage of the pulse changes the inter-brane distance from $2 \pi \mathbf{w}_{\text {in }}$ to $2 \pi \mathbf{w}_{\text {out }}$. Then the asymptotic 'in' and 'out' fields read :

$$
\mathbf{X}_{\mathrm{in}}(\sigma, \tau)=\mathbf{w}_{\mathrm{in}}(\pi-2 \sigma)+\sum_{n \neq 0} \frac{1}{n} \mathbf{a}_{n}^{(\mathrm{in})} e^{-i n \tau} \sin (n \sigma),
$$

and

$$
\mathbf{X}_{\text {out }}(\sigma, \tau)=\mathbf{b}+\mathbf{w}_{\text {out }}(\pi-2 \sigma)+\sum_{n \neq 0} \frac{1}{n} \mathbf{a}_{n}^{\text {(out) }} e^{-i n \tau} \sin (n \sigma) .
$$

Furthermore, since the problem is linear in the external fields, the special solutions are those given in the previous section, plus an extra contribution that takes care of the 
relative motion of the branes $\mathbf{Z}\left(x^{+}\right)$. Explicitly :

$$
\begin{aligned}
\delta \mathbf{X}_{\text {in }}(\sigma, \tau) & =\mathbf{F}_{\text {in }}\left(\tau-\sigma, p^{+}\right)+\mathbf{F}_{\text {in }}\left(\tau+\sigma-\pi, p^{+}\right) \\
& +\mathbf{G}_{\text {in }}\left(\tau-\sigma, p^{+}\right)-\mathbf{G}_{\text {in }}\left(\tau+\sigma-\pi, p^{+}\right),
\end{aligned}
$$

where $\mathbf{F}_{\text {in }}$ is defined as in (3.12), and

$$
\mathbf{G}_{\text {in }}(a, \lambda)=\sum_{N=0}^{\infty}\left[\mathbf{Z}(\lambda a-\lambda N \pi)-\pi \mathbf{w}_{\text {in }}\right] .
$$

Similarly

$$
\begin{aligned}
\delta \mathbf{X}_{\text {out }}(\sigma, \tau) & =\mathbf{F}_{\text {out }}\left(\tau-\sigma, p^{+}\right)+\mathbf{F}_{\text {out }}\left(\tau+\sigma-\pi, p^{+}\right) \\
& +\mathbf{G}_{\text {out }}\left(\tau-\sigma, p^{+}\right)-\mathbf{G}_{\text {out }}\left(\tau+\sigma-\pi, p^{+}\right),
\end{aligned}
$$

where $\mathbf{F}_{\text {out }}$ is given by equation (3.13), and

$$
\mathbf{G}_{\text {out }}(a, \lambda)=-\sum_{N=1}^{\infty}\left[\mathbf{Z}(\lambda a+\lambda N \pi)-\pi \mathbf{w}_{\text {out }}\right] .
$$

The new boundary conditions (4.2) are satisfied thanks to the identity : $\mathbf{G}_{\text {in }}\left(\tau, p^{+}\right)-$ $\mathbf{G}_{\text {in }}\left(\tau-\pi, p^{+}\right)=\mathbf{Z}\left(p^{+} \tau\right)$, which the reader will have no trouble to verify. Clearly the solution (4.6) reduces to the one of the previous section when $\mathbf{Z}\left(x^{+}\right)$, and hence also the sums $\mathbf{G}_{\text {in }}$ and $\mathbf{G}_{\text {out }}$, are set to zero.

It is instructive to dualize the above solution, so as to describe an open string coupled to Born-Infeld electromagnetic waves. The T-dual gauge fields are

$$
\mathbf{A} \pm \mathbf{B} \equiv\left(2 \pi \alpha^{\prime}\right)^{-1}(\mathbf{Y} \pm \mathbf{Z})
$$

The open string is charged with respect to the field $B$, and couples as a dipole to the field $A$. The classical string trajectory is readily obtained by changing the sign of the right-moving part of the transverse coordinates, $\partial_{ \pm} \mathbf{X} \rightarrow \mp \partial_{ \pm} \mathbf{X}$. This is the action of a T-duality transformation on the worldsheet [32]. In the case at hand we find $\mathbf{X}^{\prime}=\mathbf{X}_{\mathrm{in}}^{\prime}+\delta \mathbf{X}_{\mathrm{in}}^{\prime}$, with

$$
\mathbf{X}_{\mathrm{in}}^{\prime}(\sigma, \tau)=\mathbf{p}_{\text {in }} \tau-\sum_{n \neq 0} \frac{i}{n} \mathbf{a}_{n}^{(\mathrm{in})} e^{-i n \tau} \cos (n \sigma)
$$

and

$$
\begin{aligned}
\delta \mathbf{X}_{\mathrm{in}}^{\prime}(\sigma, \tau) & =\mathbf{F}_{\mathrm{in}}^{\prime}\left(\tau-\sigma, p^{+}\right)-\mathbf{F}_{\mathrm{in}}^{\prime}\left(\tau+\sigma-\pi, p^{+}\right) \\
& +\mathbf{G}_{\mathrm{in}}^{\prime}\left(\tau-\sigma, p^{+}\right)+\mathbf{G}_{\mathrm{in}}^{\prime}\left(\tau+\sigma-\pi, p^{+}\right),
\end{aligned}
$$


where

$$
\mathbf{F}_{\text {in }}^{\prime}(a, \lambda)=\pi \sum_{N=0}^{\infty}(-)^{N} \mathbf{A}(\lambda a-\lambda N \pi)
$$

and

$$
\mathbf{G}_{\text {in }}^{\prime}(a, \lambda)=\pi \sum_{N=0}^{\infty} \mathbf{B}(\lambda a-\lambda N \pi) .
$$

We have here used a gauge transformation to set $\mathbf{A}(-\infty)=\mathbf{B}(-\infty)=0$. Notice that the asymptotic brane separation $2 \pi \mathbf{w}_{\text {in }}$ has been mapped here to transverse momentum in the initial state. Equations (4.11) - (4.14) are the complete solution to the problem of an open string coupling to two parallel plane electromagnetic waves.

Before discussing further the above solution, it will be again useful to comment on its point-particle limit. The novelty with respect to our discussion in the previous section, is that a charged point particle can now accelerate in the electric field of the wave $\mathbf{B}\left(x^{+}\right)$, and emerge with a modified momentum

$$
\mathbf{p}_{\text {out }}-\mathbf{p}_{\text {in }}=-q \mathbf{B}(+\infty)
$$

This follows by integrating the equation of motion

$$
m \frac{d^{2} X^{\mu}}{d \tau^{2}}=q F^{\mu \nu} \frac{d X_{\nu}}{d \tau}
$$

where $F_{+j}=\partial_{+} B_{j}$, and $q$ is the charge of the particle that I assumed minimally coupled. Since $p^{+}$is always conserved, and $p^{-}=\left(\mathbf{p}^{2}+m^{2}\right) / 2 p^{+}$in the asymptotic regions, it follows that the momentum $p^{-}$will also generally change in the process. This possibility of net momentum transfer even in the point-particle limit is the main difference between a charged and a neutral string. Notice that for a neutral particle, the invariant momentum transfer $t=\left(p_{\text {out }}-p_{\text {in }}\right)^{2}$ is zero, because both $p^{+}$and the transverse $\mathbf{p}$ are conserved.

In the original T-dual picture of Figure 2, momentum transfer corresponds to the elongation (or contraction) of the open string, due to the net relative displacement of the D-branes. This duality has been invoked previously in [45] in order to give a 'soap-bubble' interpretation of the high-energy behaviour of the Veneziano amplitude. Note also that the above analysis can be extended easily to 'mixed' situations, such as the $\mathrm{Dp} / \mathrm{D}(\mathrm{p}+4)$ system carrying both vibration and electromagnetic waves. Rather than guessing the special solutions, as we have done here, it is however preferable to construct them directly using the Green functions on the worldsheet. This brings us naturally to the issue of first quantization, to which we will next turn our attention. 


\section{First Quantization}

The quantum mechanical evolution of the string is described by a unitary operator acting on the asymptotic incoming Fock space. This Fock space is constructed as usual, by interpreting the oscillation amplitudes $\mathbf{a}_{n}^{(i n)}$ of the incoming fields (4.4) as creation or annihilation operators. In the interaction picture, the evolution operator follows directly from the expression (2.3) for the marginal deformation,

$$
U(\tau)=T \exp \left(i \int_{-\infty}^{\tau} \frac{d \rho}{\pi} \mathcal{H}_{\mathrm{I}}(\rho)\right)
$$

where $T$ denotes time ordering, and the interaction Hamiltonian is

$$
\mathcal{H}_{\mathrm{I}}(\rho)=\tilde{\mathbf{Y}}_{\text {left }}\left(p^{+} \rho\right) \cdot \partial_{\sigma} \mathbf{X}_{\text {in }}(0, \rho)-\tilde{\mathbf{Y}}_{\text {right }}\left(p^{+} \rho\right) \cdot \partial_{\sigma} \mathbf{X}_{\mathrm{in}}(\pi, \rho) .
$$

Here the tildes signify that the past asymptotic values of the displacement vectors, i.e. $\pm \pi \mathbf{w}_{\text {in }}$ for $\mathbf{Y}_{\text {left }}$ and $\mathbf{Y}_{\text {right }}$, are subtracted away. Using the definitions (4.1), and the expansion of the incoming field (4.4), one finds

$$
\frac{1}{2} \mathcal{H}_{\mathrm{I}}(\rho)=\sum_{n \text { odd }} e^{-i n \rho} \mathbf{a}_{n}^{(\mathrm{in})} \cdot \mathbf{Y}\left(p^{+} \rho\right)+\sum_{n \text { even }} e^{-i n \rho} \mathbf{a}_{n}^{(\mathrm{in})} \cdot\left(\mathbf{Z}\left(p^{+} \rho\right)-\pi \mathbf{w}_{\text {in }}\right)
$$

where we have defined the zero mode $\mathbf{a}_{0}^{(\mathrm{in})} \equiv-2 \mathbf{w}_{\text {in }}$, and the dots denote the vector inner product. The worldsheet S-matrix, that maps the incoming states of the open string to outgoing single-string states, can be obtained as the limit of the evolution operator,

$$
S=\lim _{\tau \rightarrow \infty} U(\tau)
$$

Since the interaction is linear in the incoming fields, whose commutators are just cnumbers, we can remove the time ordering of $U(\tau)$ at the expense of introducing an overall real phase (see for instance [46]) :

$$
U(\tau)=\exp \left(i \delta_{1}\right) \times \exp \left(i \int_{-\infty}^{\tau} \frac{d \rho}{\pi} \mathcal{H}_{\mathrm{I}}(\rho)\right)
$$

where

$$
\delta_{1}=\frac{i}{2} \int_{\infty}^{\tau} \int_{\infty}^{\tau} \frac{d \rho d \rho^{\prime}}{\pi^{2}}\left[\mathcal{H}_{\mathrm{I}}(\rho), \mathcal{H}_{\mathrm{I}}\left(\rho^{\prime}\right)\right] \theta\left(\rho-\rho^{\prime}\right) .
$$

Note that $\mathcal{H}_{\mathrm{I}}(\rho)$ is a Hermitean operator, so $\delta_{1}$ is indeed a real phase. It is a function of the momentum $p^{+}$, and of the charge sector of the open string, but does not depend otherwise 
on the incoming state. For a neutral string in the adiabatic limit (see next section) this phase shift should be proportional to the time delay (3.3) . More generally, it can be evaluated explicitly, but this will not concern us any further here.

Let us instead consider the string-coordinate operators, which can be expressed in the Heisenberg representation as follows :

$$
\mathbf{X}(\tau, \sigma) \equiv \mathbf{X}_{\mathrm{in}}(\tau, \sigma)+\delta \mathbf{X}_{\mathrm{in}}(\tau, \sigma)=U(\tau)^{-1} \mathbf{X}_{\mathrm{in}}(\tau, \sigma) U(\tau)
$$

Using the equation (5.5) and the identity

$$
e^{-A} B e^{A}=B+[A, B]
$$

valid whenever the commutator $[A, B]$ is a c-number, one finds

$$
\delta \mathbf{X}_{\mathrm{in}}(\tau, \sigma)=-i \int_{-\infty}^{\tau} \frac{d \rho}{\pi}\left[\mathcal{H}(\rho), \mathbf{X}_{\mathrm{in}}(\tau, \sigma)\right] .
$$

Plugging in the expression (5.3) for the interaction Hamiltonian, and using the canonical commutation relations

$$
\left[a_{m}^{i}, a_{n}^{j}\right]=m \delta_{m+n} \delta^{i j}
$$

as well as the identities

$$
\sum_{n \text { even }} e^{i n \alpha}=\pi \sum_{m=-\infty}^{\infty} \delta(\alpha-m \pi)
$$

and

$$
\sum_{n \text { odd }} e^{i n \alpha}=\pi \sum_{m=-\infty}^{\infty}(-)^{m} \delta(\alpha-m \pi)
$$

gives precisely ${ }^{4}$ the expression (4.6) for $\delta \mathbf{X}_{\mathrm{in}}$. In fact, the above argument is an alternative, direct derivation of this expression, which can be extended easily to the general 'mixed' situations mentionned in section 4 . It is of course not surprising that the semiclassical approximation is exact, since the interaction Hamiltonian is linear.

4 The contribution of the zero mode in this calculation is subtle. It arises because in the expansion (4.4) for the quantum field, one must include the dual position operator that has a canonical commutation relation with $\mathbf{a}_{0}$. 
We concentrate now on the $S$ matrix, which describes the outgoing states of the open string long after the passage of the wave. Combining equation (5.3) with our definition (2.12) of the Fourier transforms we find :

$$
i \int_{-\infty}^{\infty} \frac{d \rho}{\pi} \mathcal{H}_{\mathrm{I}}=i h_{0}+\sum_{n \text { odd }} \frac{4 i}{n} \mathbf{a}_{n}^{(\mathrm{in})} \cdot \mathbf{y}\left(n / p^{+}\right)+\sum_{n \text { even } \neq 0} \frac{4 i}{n} \mathbf{a}_{n}^{(\mathrm{in})} \cdot \mathbf{z}\left(n / p^{+}\right)
$$

where $\mathbf{y}$ and $\mathbf{z}$ are the Fourier transforms, respectively, of $\mathbf{Y}$ and $\mathbf{Z}$, and $h_{0}$ is the contribution of the zero modes,

$$
h_{0}=2 \int_{-\infty}^{\infty} \frac{d \rho}{\pi} \mathbf{a}_{0}^{(\mathrm{in})} \cdot\left(\mathbf{Z}\left(p^{+} \rho\right)-\pi \mathbf{w}_{\text {in }}\right) .
$$

Notice that $\mathbf{a}_{0}^{(\mathrm{in})}$ is here an operator, while $\mathbf{w}_{\text {in }}$ is the asymptotic value of the wave. The integral (5.14) diverges whenever $\mathbf{w}_{\text {out }} \neq \mathbf{w}_{\text {in }}$, i.e. when the string is elongated (or contracted) by the wave. In this case $h_{0}=2\left(\mathbf{w}_{\text {out }}-\mathbf{w}_{\text {in }}\right) \cdot \hat{\mathbf{x}}_{0}$, where $\hat{\mathbf{x}}_{0}$ is the pseudoposition operator that is canonically conjugate to $\mathbf{a}_{0}^{(i n)}$. This statement can be understood easily if one considers a charged particle in an electromagnetic pulse, for which the S-matrix must obey the relation $S^{-1} \hat{\mathbf{p}}_{\text {in }} S=\mathbf{p}_{\text {out }}-\mathbf{p}_{\text {in }}$.

Let us consider finally the oscillator contributions to the S-matrix. Using the wellknown identity, valid for a c-number commutator,

$$
e^{A+B}=e^{A} e^{B} e^{[A, B] / 2}
$$

we can put the S-matrix in normal-ordered form :

$$
S=\exp \left(i \delta_{1}-\delta_{2}\right) \times: \exp \left(i \int_{-\infty}^{\infty} \frac{d \rho}{\pi} \mathcal{H}_{\mathrm{I}}(\rho)\right):
$$

where the imaginary phase $\delta_{2}$ can be extracted easily from the expression (5.13),

$$
\delta_{2}=\sum_{n=1,3, . .} \frac{8}{n}\left|\mathbf{y}\left(n / p^{+}\right)\right|^{2}+\sum_{n=2,4, . .} \frac{8}{n}\left|\mathbf{z}\left(n / p^{+}\right)\right|^{2}
$$

Equations (5.13) - (5.17) are the main result of this section. They give the exact S-matrix of the open quantum string in the background of an arbitrary plane wave.

A string initially in its ground state will emerge in the coherent superposition $S \mid p^{+}>_{\text {in }}$ after crossing the pulse. The imaginary phase $\delta_{2}$ determines the probability for the string not to be excited by the wave,

$$
\mid \text { in }<p^{+}|S| p^{+}>\left._{\text {in }}\right|^{2}=e^{-2 \delta_{2}} .
$$


Notice that the synchronized wave, $\mathbf{Y}\left(x^{+}\right)$, excites the odd frequencies of the string, while the relative wave, $\mathbf{Z}\left(x^{+}\right)$, excites the even frequencies. This, and the zero-mode contribution, are the only differences between these two types of wave. The average square mass of the outgoing string, taken initially in its ground state, is

$$
<M^{2}>=4 \mathbf{w}_{\text {out }}^{2}+32 \times\left(\sum_{n=1,3, . .}\left|\mathbf{y}\left(n / p^{+}\right)\right|^{2}+\sum_{n=2,4, . .}\left|\mathbf{z}\left(n / p^{+}\right)\right|^{2}\right) .
$$

As can be easily seen, the excitation probabilities vanish in the limit $p^{+} \rightarrow 0$, provided the shape of the background pulse is smooth (i.e. it does not contain arbitrarily high frequencies). This is the adiabatic limit, to which we will now turn our attention.

\section{Multipole Expansion}

The results of the previous section make it clear that the most relevant parameter in the problem is $\omega p^{+} \alpha^{\prime}$, where $\omega$ is the characteristic frequency of the pulse. In general a pulse will have many frequencies, possibly inside different bandwiths. If the maximal characteristic frequency is sufficiently small,

$$
\omega_{\max } p^{+} \alpha^{\prime} \ll 1
$$

the excitation probability will be practically zero. The string will surf in this case on the wave, and emerge behind it having suffered at most an adiabatic elongation or contraction. Of course, when $p^{+}=0$ the string rides the wave forever, it is indeed indistinguishable from the massless strings that make the wave.

We can study the low-frequency limit in more detail with the help of the EulerMacLaurin formula, which expresses the difference between a discrete sum and the corresponding integral :

$$
\sum_{n=0}^{\infty} \epsilon f(x-n \epsilon)=\int_{-\infty}^{x} d y f(y)+\frac{\epsilon}{2} f(x)+\sum_{r=1}^{\infty} \frac{B_{2 r}}{2 r !} \epsilon^{2 r} f^{(2 r-1)}(x) .
$$

Here $B_{n}$ are the Bernoulli numbers, $B_{2}=1 / 6, B_{4}=-1 / 30 \cdots$, and $\epsilon$ a small expansion parameter. Using this formula we can express the function $\mathbf{G}_{\text {in }}(x / \lambda, \lambda)$, given by the discrete sum (4.7), as an asymptotic power series in $\lambda$ for fixed $x$ :

$$
\mathbf{G}_{\mathrm{in}}(x / \lambda, \lambda)=\frac{1}{\pi \lambda} \int_{-\infty}^{x} d y \tilde{\mathbf{Z}}(y)+\frac{1}{2} \tilde{\mathbf{Z}}(x)+\sum_{r=1}^{\infty} \frac{B_{2 r}}{2 r !}(\pi \lambda)^{2 r-1} \mathbf{Z}^{(2 r-1)}(x),
$$


where $\tilde{\mathbf{Z}} \equiv \mathbf{Z}-\pi \mathbf{w}_{\text {in }}$ as previously defined. The function $\mathbf{F}_{\text {in }}(x / \lambda, \lambda)$ given by the alternating sum (4.8) can be expanded similarly. One uses the Euler-MacLaurin formula for the even and odd terms of the sum separately, and then performs a Taylor expansion of their difference. The first few terms of the resulting series are :

$$
\mathbf{F}_{\text {in }}(x / \lambda, \lambda)=\frac{1}{2} \mathbf{Y}(x)+\frac{\pi \lambda}{4} \mathbf{Y}^{\prime}(x)-\frac{(\pi \lambda)^{3}}{48} \mathbf{Y}^{\prime \prime \prime}(x)+\cdots
$$

with primes denoting derivatives of the function $\mathbf{Y}$. All higher-order terms are of course easy to compute, if necessary.

We want to insert these expansions in the equation (4.6) for the string coordinates. It is convenient to define $\tilde{\sigma} \equiv \pi / 2-\sigma$, so that the center of the open string is located at $\tilde{\sigma}=0$. The arguments of $\mathbf{F}_{\text {in }}$ and $\mathbf{G}_{\text {in }}$ in (4.6) are $\lambda=p^{+}$and $x=x^{+}-\tilde{\epsilon}$, where $\tilde{\epsilon}=p^{+}(\pi / 2 \mp \tilde{\sigma})$. We need therefore to further expand (6.3) and (6.4) in powers of $\tilde{\epsilon}$. After some lengthy but straightforward algebra one finds :

$$
\begin{gathered}
\mathbf{G}_{\text {in }}\left(\tau-\sigma, p^{+}\right)-\mathbf{G}_{\text {in }}\left(\tau+\sigma-\pi, p^{+}\right)= \\
=\left(\mathbf{Z}-\pi \mathbf{w}_{\text {in }}\right)(2 \tilde{\sigma} / \pi)+\mathbf{Z}^{\prime \prime}\left(p^{+}\right)^{2}\left(\tilde{\sigma}^{3} / 3 \pi-\pi \tilde{\sigma} / 12\right)+o\left(\mathbf{Z}^{(4)}\left(p^{+}\right)^{4}\right)
\end{gathered}
$$

and

$$
\begin{gathered}
\mathbf{F}_{\mathrm{in}}\left(\tau-\sigma, p^{+}\right)+\mathbf{F}_{\mathrm{in}}\left(\tau+\sigma-\pi, p^{+}\right)= \\
=\mathbf{Y}+\mathbf{Y}^{\prime \prime}\left(p^{+}\right)^{2}\left(\tilde{\sigma}^{2} / 2-\pi^{2} / 8\right)+o\left(\mathbf{Y}^{(4)}\left(p^{+}\right)^{4}\right)
\end{gathered}
$$

The functions $\mathbf{Y}$ and $\mathbf{Z}$ and all their derivatives are evaluated in the above expressions at $x^{+}=p^{+} \tau$. Putting together everything, we arrive at the following small- $p^{+}$expansion of the transverse string coordinates, expressed as functions of the lightcone time $x^{+}$and of the string parameter $\tilde{\sigma}$ :

$$
\delta \mathbf{X}_{\mathrm{in}}=\mathbf{Y}+\left(\mathbf{Z}-\pi \mathbf{w}_{\mathrm{in}}\right) \frac{2 \tilde{\sigma}}{\pi}+\left(p^{+}\right)^{2}\left(\mathbf{Y}^{\prime \prime}+\frac{2 \tilde{\sigma}}{3 \pi} \mathbf{Z}^{\prime \prime}\right)\left(\frac{\tilde{\sigma}^{2}}{2}-\frac{\pi^{2}}{8}\right)+o\left(\left(p^{+}\right)^{4}\right)
$$

As a check of the above result, notice that it obeys the free worldsheet equation, $\left(\partial_{\tau}^{2}-\partial_{\tilde{\sigma}}^{2}\right) \delta \mathbf{X}_{\text {in }}=0$, and the boundary conditions $\mathbf{X}_{\text {in }}+\delta \mathbf{X}_{\text {in }}=\mathbf{Y} \pm \mathbf{Z}$ at $\tilde{\sigma}= \pm \pi / 2$. Allowed terms in the expansion respect, furthermore, two discrete symmetries : (a) invariance under orientation flip $(\tilde{\sigma} \rightarrow-\tilde{\sigma})$ combined with an exchange of the two D-branes $(\mathbf{Z} \rightarrow-\mathbf{Z})$, and (b) invariance under time reversal on the worldsheet $(\tau \rightarrow-\tau)$ combined with the spacetime reflection $x^{ \pm} \rightarrow-x^{ \pm}$. It is this second symmetry that explains the absence of odd-derivative terms in (6.7). We can in fact use these discrete symmetries, together with 
the linearity of the response, the free-wave equation and the boundary conditions, to give an alternative systematic derivation of the multipole expansion.

Let us evaluate the series (6.7) at a time $x^{+}$, long after the passage of the pulse. Since the derivatives of $\mathbf{Y}$ and $\mathbf{Z}$ go to zero, only the first two terms of the series survive. They describe an open string which emerges from the wave in its initial state of oscillation, given by $\mathbf{X}_{\mathrm{in}}$. The linear term in $\delta \mathbf{X}_{\text {in }}$ corresponds to an adiabatic elongation or contraction, as expected. The process of string excitation (or the inverse process) is not, in other words, visible at any finite order of the small- $p^{+}$expansion.

Before concluding this section, it is instructive to consider also the T-dual problem of an open string in an electromagnetic wave. The classical trajectory, eqs. (4.11) and (4.12), can be expanded easily with the help of the Euler-Maclaurin formulae (6.3) and (6.4). The first few non-trivial terms are :

$$
\delta \mathbf{X}_{\mathrm{in}}^{\prime}=2 \int_{-\infty}^{x^{+}} \mathbf{B}(y) d y+p^{+} \mathbf{B}^{\prime}\left(\tilde{\sigma}^{2}-\pi^{2} / 4\right)+p^{+} \mathbf{A}^{\prime} \pi \tilde{\sigma}+o\left(\left(p^{+}\right)^{5}\right)
$$

The first term comes from the monopole coupling to the gauge field $\mathbf{B}$, while the third term is due to the leading, dipole coupling to the gauge field $\mathbf{A}$. To check this, note that a pair of opposite unit charges have the following dipole coupling to the electromagnetic field, $\delta \mathcal{L}=F_{\mu \nu} r^{\mu} \dot{X}^{\nu}+\cdots$, where $X$ and $r$ are the center-of-mass and relative positions. For a plane-fronted wave, and in lightcone gauge, this gives $\delta \mathcal{L}=-p^{+} \mathbf{A}^{\prime} \cdot \mathbf{r}+\cdots$. If the charges are connected by a string, there is furthermore a tension energy equal to $\mathbf{r} \cdot \mathbf{r} /\left(4 \pi^{2} \alpha^{\prime}\right)$. The minimum of the energy is thus at $\mathbf{r} \equiv \delta \mathbf{X}_{\mathrm{in}}^{\prime}(\pi)-\delta \mathbf{X}_{\mathrm{in}}^{\prime}(0)=p^{+} \mathbf{A}^{\prime} \pi^{2}$, in full agreement with the third term in (6.8).

\section{Shock waves, Boomerangs and Other Beasts}

The failure of the multipole expansion to describe the process of string excitation is most evident in the limiting case of a $\delta$-function pulse. Consider a pulse propagating entirely on the left D-string in Figure 2. This corresponds to the choice

$$
\mathbf{Y}\left(x^{+}\right)=\mathbf{Z}\left(x^{+}\right)-\pi \mathbf{w}_{\text {in }}=\frac{1}{2} \mathbf{C} \delta\left(x^{+}\right)
$$

with $\mathbf{C}$ an arbitrary polarization vector. We assume also, for definiteness, that the open string is initially in its ground state and at rest, so that its energy equals tension times length, $p^{+}=p^{-}=\sqrt{2}\left|\mathbf{w}_{\mathrm{in}}\right|$. Its classical worldsheet at later times is given by the 
right-hand-side of equation (4.6). This is a sum of $\delta$-functions, which in the time interval $N \pi p^{+} \leq x^{+} \leq(N+1) \pi p^{+}$is equal to :

$$
\delta \mathbf{X}_{\text {in }}=\left\{\begin{array}{cc}
0 & \text { for } N<0 \\
\mathbf{C} \delta\left(x^{+}-p^{+} \sigma-N p^{+} \pi\right) & \text { for } N \geq 0 \text { even } \\
-\mathbf{C ~} \delta\left(x^{+}-p^{+}(\pi-\sigma)-N p^{+} \pi\right) & \text { for } N>0 \text { odd } .
\end{array}\right.
$$

This time-dependence can be visualized in Figure 3. The primary pulse propagates initially on the left D-string, and reaches the endpoint of the fundamental string at time $x^{+}=0$. There it sends a clone ripple down the open string, while continuing on its way unchanged. This sounds like a violation of energy, but recall that in the zero-string-coupling limit, in which we here work, the D-string tension is infinite and back reaction can thus be neglected. After the D-brane ripple has left the scene, its clone on the fundamental string keeps bouncing back and forth, fliping sign after every reflection. Clearly, this behaviour cannot be captured by the multipole expansion, which 'predicts' that the open string must return to its ground state adiabatically, after the pulse has left.

Two remarks are here in order. First, one may wonder how the pulse can be transmitted when the polarization vector $\mathbf{C}$ points in the direction of the stretched string. The answer is that in the lightcone gauge the reparametrization invariance is completely fixed,

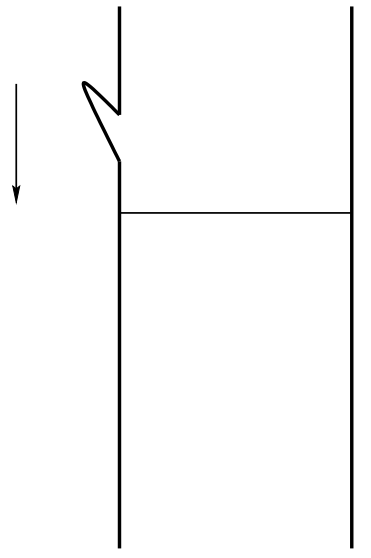

(a)

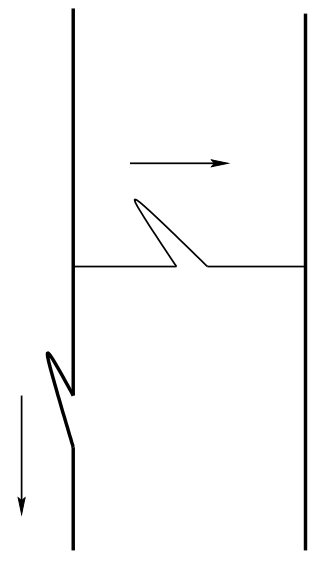

(b)

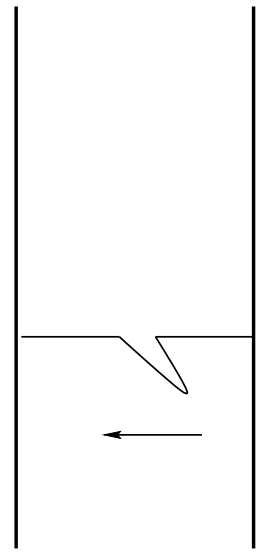

(c)

Fig. 3: Three snapshots of the stretched open string (a) before being hit on the left by the pulse, (b) immediately after, and (c) after the secondary pulse has been reflected once at the right endpoint. The string's recoil in the direction of the pulse is not accurately depicted by this drawing. In the second-quantized theory, open and/or closed strings will be radiated away in the process. 
so longitudinal oscillations are physical. The second, more important remark, is that for a primary wave of arbitrary shape, the secondary ripples on the open string are given by superposing the solutions (7.2) . This follows from the linearity of the problem, and the fact that any temporal profile is a superposition of $\delta$-function pulses like (7.1). Thus, any smooth pulse of sufficiently short duration will give the same qualitative behaviour as illustrated in Figure 3. Pulses of longer duration, on the other hand, will lead to constructive or destructive interference between the disturbances entering the string from the left, and those bouncing back and forth between its endpoints.

It should be stressed that although the transverse coordinates $\mathbf{X}$ respond linearly to the wave, the same is not the case for the light-cone coordinate $X^{-}$. This is fixed by the Virasoro condition (3.6), which implies that $X^{-}$is a quadratic function of the primary pulse. Note in particular that the string recoils after being hit by the pulse, as can be deduced from the expression for the center-of-mass momentum

$$
p^{-}(\tau)=\frac{1}{2 \pi p^{+}} \int_{0}^{\pi} d \sigma\left(\partial_{\tau} \mathbf{X} \cdot \partial_{\tau} \mathbf{X}+\partial_{\sigma} \mathbf{X} \cdot \partial_{\sigma} \mathbf{X}\right)
$$

The net recoil, $\delta p^{-}=p_{\text {out }}^{-}-p_{\text {in }}^{-}$, diverges for a $\delta$-function pulse, and so does the expectation value (5.19) of the mass squared operator in the quantum problem. This is not surprising, since the wave carries infinite energy and momentum in this limit. For a smooth but sufficiently short pulse (for which the interference terms can be neglected), one finds

$$
\delta p^{-} \simeq \frac{2 T_{F}}{T_{D}} P_{\text {pulse }}^{-}
$$

where $T_{F}=\left(2 \pi \alpha^{\prime}\right)^{-1}$ is the tension of the fundamental string, and we have used equation (2.11) . Thus the outgoing string carries away a fixed fraction of the incident $p^{-}$momentum in this limit. Note that since $T_{D} \sim 1 / g_{s}$, this fraction is vanishingly small at weak string coupling and the back reaction can be indeed neglected.

The effects of an incident pulse with a cusp (or kink) can be analyzed similarly with the help of equations (5.17) and (5.19). If $\mathbf{Y}$, or $\mathbf{Z}$, behave like $\left|x^{+}\right|^{\beta}$ near the cusp, then their Fourier transforms $\mathbf{y}(\omega)$, or $\mathbf{z}(\omega)$, vary at high frequencies like $|\omega|^{-\beta}$. Since $\delta_{2}$ converges for all (positive) values of $\beta$, the string has a finite probability to traverse the pulse and remain in its ground state. The average mass squared of the outgoing string would, on the other hand, diverge for $\beta \leq 1 / 2$. This is precisely the range of $\beta$ in which the integral (2.11) for the energy and momentum of the D-brane also diverges. We conclude that cusps are acceptable singularities, as long as they transport finite energy and momentum. 
Having discussed the high-frequency components of the wave, let me next comment on other potential sources of divergent behaviour. One such source is of course a highly monochromatic wave, which can produce resonnant excitation of the open string. As an example, consider the following helical D-string :

$$
Y^{2}+i Y^{3}=C e^{i \omega_{0} x^{+}} \Longleftrightarrow \mathrm{y}^{2}+i \mathrm{y}^{3}=C \omega \delta\left(\omega-\omega_{0}\right)
$$

The $\delta$-function makes a divergent contribution to (5.19), whose origin is however easy to isolate. Indeed, using Fermi's golden rule for a wave train of total duration $T$, and assuming that the incoming open string has a smooth wavefunction $\psi\left(p^{+}\right)$in momentum space (normalized so that $\int d p^{1}\left|\psi\left(p^{+}\right)\right|^{2}=1$ ) we find :

$$
<M^{2}>=\frac{8 T}{\pi} C^{2} \omega_{0}^{2} \times \sum_{n \text { odd }}\left|\psi\left(n / \omega_{0}\right)\right|^{2}
$$

Thus, for an incoming open-string wavepacket, the divergence is regularized by the finite duration of the pulse. Notice that the energy drained out of the wave by the string grows only like the square root of time.

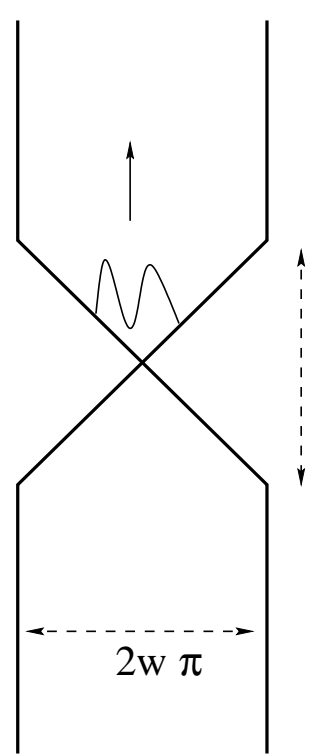

(a)

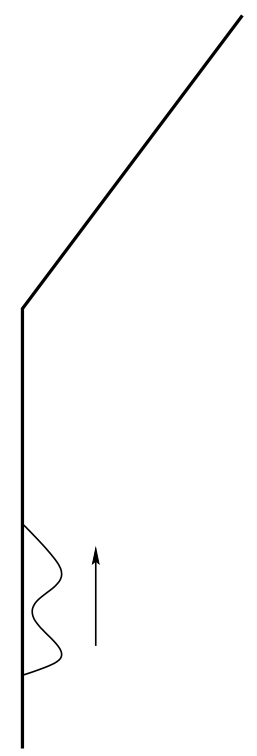

(b)

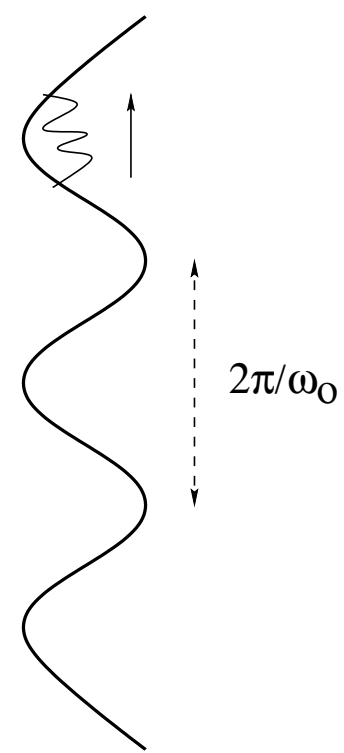

(c)

Fig. 4: Three configurations discussed in the main text : (a) regularized null D-brane scissors, (b) a boomerang, and (c) a monochromatic wave. 
A different type of infrared problem occurs for the null D-brane scissors discussed in ref. [20]. In this configuration $\mathbf{Z}$ grows linearly with $x^{+}$, leading to an infinite adiabatic elongation of the stretched strings. As a result, there are no normalizable states for the zero modes except when $p^{+}=0$ [20]. To isolate this problem, let us regularize the large-time behaviour of the profile function. We can choose, for instance, a piecewise-linear profile like the one illustrated in Figure 4 :

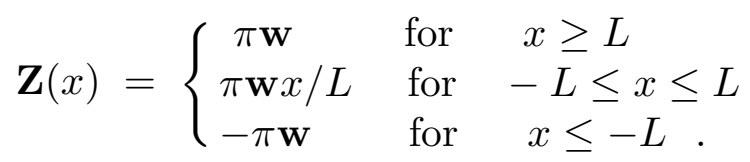

Its Fourier transform can be easily calculated with the result :

$$
\mathbf{z}(\omega)=-i \mathbf{w} \frac{\sin (\omega L)}{\omega L}
$$

The $1 / \omega$ behaviour at high frequencies is characteristic of the abrupt kinks at $x= \pm L$. The basic point, however, here is that the sum (5.19), which is a measure of the string's excitation, can be made arbitrarily small for large enough $L / p^{+} \alpha^{\prime}$. This shows that the null brane intersection does not, per se, introduce any singularity, at least at the level of first-quantized string theory.

The configuration (7.7) is an open string analog of the parabolic orbifold 'cosmology' [4][14], in which the 'universe' expansion comes to a stop. The null brane intersection plays the role of the Big Bang/Big Crunch singularity, while stretched open strings are analogous to twisted closed strings. The intersection point (like the null orbifold singularity) can be regularized by an extra shift in one of the spectator dimensions. It was argued in ref. [20] that string collisions near the intersection point can catalyze a large backreaction, resulting possibly in an intercommutation of the D-strings. This would allow any stretched open strings, formed during the collision process, to escape with finite energy to infinity. Indeed, after intercommutation each D-string has a boomerang shape, $\mathrm{Y}^{2}\left(x^{+}\right)=C x^{+} \theta\left(x^{+}\right)$, as illustrated in Figure 4b. An open string moving on such a D-brane will pick up momentum $p^{2}=C p^{+}$while making the turn at $x^{+}=0$. The string's energy in the outgoing state will depend also on its oscillation mass, but as the reader can easily verify, this stays finite for finite $C$ (corresponding to a boomerang making an oblique angle).

A conceptual difficulty from the point of view of the open string, is that the process of brane intercommutation looks, superficially at least, non-causal. The string only starts stretching after it has crossed the intersection point, by which time it is too late to backreact on it. It would be very interesting to see how the issue is resolved in the full secondquantized string theory. 


\section{From Lasers to Gravitational Waves}

The question we should finally address is whether the coherent excitation of strings, discussed in this paper, can occur in a real-world setting. There are three kinds of relativistic strings in nature : QCD strings, fundamental strings, and cosmic strings. ${ }^{5}$ The fundamental and QCD strings are quantum mechanical, while cosmic strings have macroscopic sizes and are to a good approximation classical. Strong coherent radiation fields, on the other hand, are either electromagnetic or gravitational. As we have seen, the necessary condition for string excitation is

$$
\omega_{0} p^{+} \geq T
$$

where $\omega_{0}$ is the characteristic frequency of the wave, and $T$ and $p^{+}$are, respectively, the tension and lightcone momentum of the incident string. The first question is, therefore : in what physical settings can condition (8.1) be satisfied?

The highest-frequency coherent electromagnetic fields are produced by lasers, which operate at or near the optical spectrum. Although coherent stimulated emission in the soft X-ray region $\sim 10-15 \mathrm{~nm}$ has been apparently reported (see for instance [47]) most lasers have wavelengths larger than a few hundred nanometers. This corresponds to frequencies $\sim 4-6 \mathrm{eV}$ at most. The Regge slope of QCD strings, on the other hand, is $\alpha^{\prime}=$ $(2 \pi T)^{-1} \sim(\mathrm{GeV})^{-2}$. Coherent excitation of hadrons in a laser beam is, therefore, possible for momenta $p^{+} \geq 10^{8} \mathrm{GeV}$. This is beyond the reach of accelerators, but within the upper end of the cosmic-ray spectrum. The flux of cosmic rays at these energies is however exceedingly low, roughly one event per $m^{2} \times$ year [48]. Since the typical size of a laser beam is in the $\mu \mathrm{m}$ range, the rate of events if one placed a laser in outer space would still be ridiculously small. Note, however, that since the cosmic-ray flux rises very rapidly with decreasing energy (roughly by a factor of $10^{3}$ for each energy decade) a hard X-ray or $\gamma$-ray laser would fare much better!

The tension of fundamental open strings is at least six orders of magnitude above the tension of QCD strings, and probably closer to the Planck scale. This pushes the required momentum $p^{+}$above $\sim 10^{14} \mathrm{GeV}$, far above the GZK cutoff for cosmic rays.

This brings us to the third possibility, namely cosmic strings [27]. Their tension in realistic particle-physics models can range anywhere from the electroweak to the grandunification scale. Strings formed during a phase tansition in the early universe stretch, on

5 To be sure, the last two kinds have yet to be experimentally detected! 
the other hand, over typical distances $r_{0}$ of astronomical size. Since $p^{+} \sim T r_{0}$, the relevant criterion for their excitation is

$$
\omega_{0} r_{0} \geq 1
$$

This puts essentially no restriction on either electromagnetic or gravitational waves.

The discussion in this paper was, to be sure, about open strings coupling to a plane wave through their endpoints. Strictly speaking, this can be realized by 'necklace' strings, in which magnetic monopoles play the role of the beads in a necklace [27]. More generally, however, one may expect that if the wavefront size is much smaller than $r_{0}$, so that the wave hits the string in a relatively localized region, the results of this paper could be carried over. If this were true, then a large cosmic string passing near the source of a strong gravitational wave, could transmit a clone of this gravitational burst over an astronomically large distance (as in Figure 3). Furthermore, since propagation on the string is one-dimensional, the amplitude of the clone wave would stay constant, while the amplitude of the primary burst in the bulk of space would decrease as the inverse of the distance from the source. This teleportation of the signal might be relevant in searches for gravitational radiation. ${ }^{6}$ The question is presently being studied.

Let me finally point out that cosmic string networks are disfavoured as seeds of largescale anistropies, mainly because their non-linear evolution tends to produce incoherent primordial fluctuations [51][52][53]. ${ }^{7}$ It is worth investigating whether the simple model discussed here could have any bearing on this issue.

Acknowledgements: I thank M. Blau, T. Damour, E. Floratos, J. Iliopoulos, P. Mora, G. Papadopoulos, B. Pioline, R. Russo and F. Zwirner for useful conversations. This research was supported in part by the European IHP Networks 'Superstring Theory' (HPRN-CT-2000-00122) and 'The Quantum Structure of Spacetime' (HPRN-CT-200000131).

6 For a review of gravitational wave detection see [49]. The string will ultimately release its energy either by radiating gravitationally, or by splitting off small loops of string - see for instance [27][50] and references therein.

7 Though current CMB data is still, apparently, compatible with a sizable component of cosmicstring seeds [54], and there is also scepticism concerning the validity of the calculations [55]. 


\section{References}

[1] D. Amati and C. Klimcik, "Strings In A Shock Wave Background And Generation Of Curved Geometry From Flat Space String Theory," Phys. Lett. B 210, 92 (1988).

[2] H. J. de Vega and N. Sanchez, "Quantum String Propagation Through Gravitational Shock Waves," Phys. Lett. B 244, 215 (1990) ; "Quantum String Scattering In The Aichelburg-Sexl Geometry," Nucl. Phys. B 317, 706 (1989).

[3] G. T. Horowitz and A. R. Steif, "Space-Time Singularities In String Theory," Phys. Rev. Lett. 64, 260 (1990) ; "Strings In Strong Gravitational Fields," Phys. Rev. D 42, 1950 (1990).

[4] G. T. Horowitz and A. R. Steif, "Singular String Solutions With Nonsingular Initial Data," Phys. Lett. B 258, 91 (1991).

[5] E. Kiritsis and C. Kounnas, "String Propagation In Gravitational Wave Backgrounds," Phys. Lett. B 320, 264 (1994) [Addendum-ibid. B 325, 536 (1994)] arXiv:hepth/9310202 ; E. Kiritsis, C. Kounnas and D. Lust, "Superstring gravitational wave backgrounds with space-time supersymmetry," Phys. Lett. B 331, 321 (1994) arXiv:hep-th/9404114.

[6] R. R. Metsaev, "Type IIB Green-Schwarz superstring in plane wave Ramond-Ramond background," Nucl. Phys. B 625, 70 (2002) arXiv:hep-th/0112044.

[7] D. Berenstein, J. M. Maldacena and H. Nastase, "Strings in flat space and pp waves from N = 4 super Yang Mills," JHEP 0204, 013 (2002) arXiv:hep-th/0202021.

[8] R. R. Metsaev and A. A. Tseytlin, "Exactly solvable model of superstring in plane wave Ramond-Ramond background," Phys. Rev. D 65, 126004 (2002) arXiv:hepth/0202109].

[9] A. Dabholkar and S. Parvizi, "Dp branes in pp-wave background," Nucl. Phys. B 641, 223 (2002) arXiv:hep-th/0203231.

[10] D. Berenstein, E. Gava, J. M. Maldacena, K. S. Narain and H. Nastase, "Open strings on plane waves and their Yang-Mills duals," arXiv:hep-th/0203249.

[11] E. Kiritsis and B. Pioline, "Strings in homogeneous gravitational waves and null holography," JHEP 0208, 048 (2002) arXiv:hep-th/0204004.

[12] A. Kumar, R. R. Nayak and Sanjay, "D-brane solutions in pp-wave background," Phys. Lett. B 541, 183 (2002) arXiv:hep-th/0204025.

[13] K. Skenderis and M. Taylor, "Branes in AdS and pp-wave spacetimes," JHEP 0206, 025 (2002) arXiv:hep-th/0204054 ; "Open strings in the plane wave background. I: Quantization and symmetries," arXiv:hep-th/0211011.

[14] H. Liu, G. Moore and N. Seiberg, "Strings in a time-dependent orbifold," JHEP 0206, 045 (2002) arXiv:hep-th/0204168 ; "Strings in time-dependent orbifolds," JHEP 0210, 031 (2002) arXiv:hep-th/0206182. 
[15] O. Bergman, M. R. Gaberdiel and M. B. Green, "D-brane interactions in type IIB plane-wave background," arXiv:hep-th/0205183.

[16] M. Fabinger and J. McGreevy, "On Smooth Time-Dependent Orbifolds and Null Singularities," arXiv:hep-th/0206196.

[17] J. Maldacena and L. Maoz, "Strings on pp-waves and massive two dimensional field theories," arXiv:hep-th/0207284.

[18] P. Bain, K. Peeters and M. Zamaklar, "D-branes in a plane wave from covariant open strings," arXiv:hep-th/0208038.

[19] H. Fuji, K. Ito and Y. Sekino, "Penrose limit and string theories on various brane backgrounds," JHEP 0211, 005 (2002) arXiv:hep-th/0209004.

[20] C. Bachas and C. Hull, "Null brane intersections," arXiv:hep-th/0210269.

[21] J. F. Morales, "String theory on Dp-plane waves," arXiv:hep-th/0210229.

[22] R. C. Myers and D. J. Winters, "From D - anti-D pairs to branes in motion," arXiv:hep-th/0211042.

[23] M. R. Gaberdiel and M. B. Green, "The D-instanton and other supersymmetric Dbranes in IIB plane-wave string theory," arXiv:hep-th/0211122.

[24] K. Okuyama, "D-branes on the null-brane," arXiv:hep-th/0211218.

[25] G. Papadopoulos, J. G. Russo and A. A. Tseytlin, "Solvable model of strings in a time-dependent plane-wave background," arXiv:hep-th/0211289.

[26] D. Amati, M. Ciafaloni and G. Veneziano, "Superstring Collisions At Planckian Energies," Phys. Lett. B 197, 81 (1987) ; "Classical And Quantum Gravity Effects From Planckian Energy Superstring Collisions," Int. J. Mod. Phys. A 3, 1615 (1988).

[27] A. Vilenkin and E. P. S. Shellard, "Cosmic Strings and Other Topological defects," Cambridge University Press 1994.

[28] J. B. Griffiths, "Colliding Plane Waves in General Relativity," Oxford University Press, 1991 .

[29] M. Blau, J. Figueroa-O'Farrill, C. Hull and G. Papadopoulos, "A new maximally supersymmetric background of IIB superstring theory," JHEP 0201, 047 (2002) arXiv:hep-th/0110242 ; "Penrose limits and maximal supersymmetry," Class. Quant. Grav. 19, L87 (2002) arXiv:hep-th/0201081.

[30] C. P. Bachas, P. Bain and M. B. Green, "Curvature terms in D-brane actions and their M-theory origin," JHEP 9905, 011 (1999) arXiv:hep-th/9903210.

[31] A. Fotopoulos, "On (alpha')**2 corrections to the D-brane action for non-geodesic world-volume embeddings," JHEP 0109, 005 (2001) arXiv:hep-th/0104146.

[32] C. P. Bachas, "Lectures on D-branes," arXiv:hep-th/9806199.

[33] A. Dabholkar, J. P. Gauntlett, J. A. Harvey and D. Waldram, "Strings as Solitons and Black Holes as Strings," Nucl. Phys. B 474, 85 (1996) arXiv:hep-th/9511053. 
[34] D. Mateos and P. K. Townsend, Phys. Rev. Lett. 87, 011602 (2001) arXiv:hepth/0103030]; D. Mateos, S. Ng and P. K. Townsend, "Supercurves," Phys. Lett. B 538, 366 (2002) arXiv:hep-th/0204062.

[35] J. H. Cho and P. Oh, "Super D-helix," Phys. Rev. D 64, 106010 (2001) arXiv:hepth/0105095).

[36] O. Lunin and S. D. Mathur, "Metric of the multiply wound rotating string," Nucl. Phys. B 610, 49 (2001) arXiv:hep-th/0105136.

[37] Y. Hyakutake and N. Ohta, "Supertubes and supercurves from M-ribbons," Phys. Lett. B 539, 153 (2002) arXiv:hep-th/0204161.

[38] P. C. Aischelburg and R. U. Sexl, Gen. Rev. Grav. 2, 303 (1971).

[39] G. 't Hooft, "Graviton Dominance In Ultrahigh-Energy Scattering," Phys. Lett. B 198, 61 (1987).

[40] Ya. B. Zel'dovich and A. G. Polnarev, Astron. Zh. 51, 30 (1974) [SoV. Astron. 18, 17 (1974)]; V. B. Braginsky and L. P. Grishchuk, Zh. Eksp. Teor. Fiz. 89, 744 (1985) [Sov. Phys. JETP 62, 427 (1986)]; V. B. Braginsky and K. S. Thorne, Nature (London) 327, 123 (1987).

[41] D. Christodoulou, "Nonlinear Nature Of Gravitation And Gravitational Wave Experiments," Phys. Rev. Lett. 67, 1486 (1991).

[42] A. Abouelsaood, C. G. Callan, C. R. Nappi and S. A. Yost, "Open Strings In Background Gauge Fields," Nucl. Phys. B 280, 599 (1987).

[43] C. Bachas and M. Porrati, "Pair Creation Of Open Strings In An Electric Field," Phys. Lett. B 296, 77 (1992) arXiv:hep-th/9209032.

[44] J. Ambjorn, Y. M. Makeenko, G. W. Semenoff and R. J. Szabo, "String theory in electromagnetic fields," arXiv:hep-th/0012092.

[45] C. Bachas and B. Pioline, "High-energy scattering on distant branes," JHEP 9912, 004 (1999) arXiv:hep-th/9909171.

[46] C. Itzykson and J.-B. Zuber, "Quantum Field Theory," McGraw-Hill (1980).

[47] P. W. Milonni and J. H. Eberly, "Lasers", Wiley, 1988.

[48] T. K. Gaisser and T. Stanev, "Cosmic Rays," Phys. Rev. D 66, 010001 (2002).

[49] K. S. Thorne, "Gravitational Radiation: A New Window Onto the Universe," arXiv:grqc/9704042.

[50] T. Damour and A. Vilenkin, "Gravitational wave bursts from cosmic strings," Phys. Rev. Lett. 85, 3761 (2000) arXiv:gr-qc/0004075] ; "Gravitational wave bursts from cusps and kinks on cosmic strings," Phys. Rev. D 64, 064008 (2001) arXiv:grqc/0104026.

[51] J. Magueijo, A. Albrecht, P. Ferreira and D. Coulson, "The structure of Doppler peaks induced by active perturbations," Phys. Rev. D 54, 3727 (1996) arXiv:astroph/9605047. 
[52] G. R. Vincent, M. Hindmarsh and M. Sakellariadou, "Correlations in cosmic string networks," Phys. Rev. D 55, 573 (1997) [arXiv:astro-ph/9606137.

[53] A. Riazuelo and N. Deruelle, Annalen Phys. 9, 288 (2000) [arXiv:gr-qc/0005024.

[54] F. R. Bouchet, P. Peter, A. Riazuelo and M. Sakellariadou, "Is there evidence for topological defects in the BOOMERANG data?," Phys. Rev. D 65, 021301 (2002) arXiv:astro-ph/0005022.

[55] A. Riazuelo, N. Deruelle and P. Peter, "Topological defects and CMB anisotropies: Are the predictions reliable?," Phys. Rev. D 61, 123504 (2000) arXiv:astro-ph/9910290. 